\title{
BMJ Open Associations between gestational diabetes mellitus and the neurodevelopment of offspring from 1 month to 72 months: study protocol for a cohort study
}

\author{
Chao Li (D) , ${ }^{1,2}$ Ping Zhou, ${ }^{1}$ Yixi Cai, ${ }^{2}$ Bin Peng, ${ }^{3}$ Yongfang Liu, ${ }^{1}$ Ting Yang, ${ }^{1}$ \\ Yinying $\mathrm{Li}^{2}$ Yirong Hu, ${ }^{4}$ Yajun $\mathrm{Fu},{ }^{5}$ Zhenming Wang, ${ }^{5}$ Hong Peng, ${ }^{2}$ Yue Zhang, ${ }^{2}$ \\ Jie Chen, ${ }^{1}$ Tingyu Li, ${ }^{1}$ Li Chen (1) ${ }^{1}$
}

To cite: Li C, Zhou P, Cai Y, et al. Associations between gestational diabetes mellitus and the neurodevelopment of offspring from 1 month to 72 months: study protocol for a cohort study. BMJ Open 2020;10:e040305. doi:10.1136/ bmjopen-2020-040305

- Prepublication history for this paper is available online. To view these files, please visit the journal online (http://dx.doi org/10.1136/bmjopen-2020040305).

Received 10 May 2020 Revised 11 August 2020 Accepted 23 October 2020

Check for updates

(C) Author(s) (or their employer(s)) 2020. Re-use permitted under CC BY-NC. No commercial re-use. See rights and permissions. Published by BMJ.

For numbered affiliations see end of article.

Correspondence to

Dr Li Chen;

chenli@cqmu.edu.cn

\section{ABSTRACT}

Introduction Gestational diabetes mellitus (GDM) is a common gestational disease and an important global public health problem. GDM may affect the short-term and long-term health of offspring, but the associations between GDM and the neurodevelopment of offspring of mothers with GDM (OGDM) are still unclear, and studies based on the Chinese population are lacking. We aim to determine the associations between GDM and the neurodevelopment of OGDM by studying a cohort of OGDM and offspring of non-GDM mothers.

Methods and analysis The single-centre prospective cohort study is being conducted in China over 7 years. A total of 490 OGDM (GDM group) and 490 fromof healthy mothers (control group) will be enrolled during the same period. Baseline characteristics, neuropsychological development scores and clinical data at specific time points (at $0,1,3,6,12,24,36,48,60$ and 72 months old) will be collected from the children in both groups until the age of 6 years. The associations between GDM and the neurodevelopment of OGDM from infancy to preschool age will be analysed using a multiple linear regression model adjusted for confounders. In addition, we will compare longitudinal data to further assess the effects of GDM on neurodevelopmental trajectories.

Ethics and dissemination The study has been approved by the Ethics Committee of the Children's Hospital of Chongqing Medical University (Approval Number: (2019) Institutional Review Board (IRB) (STUDY) No. 85). The findings of this study will be disseminated through open access journals, peer-reviewed journals and scientific meetings.

Trial registration number NCT03997396.

\section{BACKGROUND}

Gestational diabetes mellitus (GDM), which is defined as abnormal glucose tolerance to varying degrees during pregnancy, is a common gestational disease that substantially influences children's health and has become an important public health problem. ${ }^{1}$ The

\section{Strengths and limitations of this study}

- This single-centre prospective cohort study aims to identify the associations between gestational diabetes mellitus (GDM) and the neurodevelopment of offspring of mothers with GDM from infancy to preschool age.

- This study analyses the effects of GDM on neurodevelopmental trajectories through a longitudinal comparison.

- To improve the follow-up rate, a detailed quality control plan is formulated.

- To eliminate confounders, comprehensive data are collected.

- If the associations between GDM and the neurodevelopment of offspring are not clarified due to insufficient sample size, we will expand the sample size or carry out multicentre studies.

prevalence of GDM has been reported to be $16.2 \%$ worldwide in 2017 , and the prevalence in China is approximately $14.8 \%$ in the same year. ${ }^{23}$ With the implementation of the second-child policy in our country, the number of older parturient women is gradually increasing. The prevalence of overweight and obesity caused by lifestyle changes is also increasing among parturient women. Therefore, the incidence of GDM in China is expected to increase.

The developmental origins of health and disease theory posits that environmental factors in the early stage of life, such as maternal nutrition and disease, may affect the risk of non-communicable diseases in adulthood. ${ }^{4}$ Furthermore, maternal nutrition and disease may also affect the health and development of infants and children and may even be transmitted intergenerationally. ${ }^{5}$ In 
recent years, increasing evidence has demonstrated that GDM has short-term effects on the health of offspring, resulting in conditions such as foetal macrosomia, neonatal hypoglycaemia and polycythaemia, ${ }^{6}$ and some studies have also suggested that GDM may increase the risk of overweight or obesity for the offspring of mothers with GDM (OGDM), ${ }^{7-9}$ while few studies have investigated the long-term effects on the neurodevelopment of offspring; moreover, reliable, long-term studies and firsthand data based on the Chinese population are lacking. Conclusions regarding these effects are conflicting (positive, inconclusive, negative), and no firm conclusions can be drawn from the limited data available. A birth cohort study in India has reported that some cognitive scores of OGDM are higher than those of controls. ${ }^{10}$ In contrast, some studies have found negative associations between GDM and the neurodevelopment of OGDM. A large epidemiological study performed with 1.3 million adolescents in Sweden has showed that the school grades in four subjects of OGDM are lower at 16 years of age. ${ }^{11}$ In the Diabetes in Pregnancy Study in Japan, OGDM have significantly poorer cognitive function at 3 years of age than offspring of non-GDM mothers. ${ }^{12}$ In addition, some studies have reported inconclusive associations. DeBoer $e t$ $a l$ has found that OGDM have differences on the mental development index scale, but no difference on the psychomotor developmental index scale at 1 year of age compared with the control group. ${ }^{13}$ A study in Southern California has showed that GDM is generally not associated with a risk of Attention deficit and hyperactivity disorder (ADHD) for offspring while OGDM requiring antidiabetic medications have a considerably greater ADHD risk than OGDM who do not require antidiabetic medications. ${ }^{14}$ However, these results were confounded by factors such as family income, prepregnancy body mass index (BMI), and smoking and drinking habits. ${ }^{15}$ These cross-sectional studies have found different associations between GDM and the neurodevelopment of OGDM, although the effects of GDM on the neurodevelopmental trajectories of offspring remains unclear.

Therefore, we must urgently establish a prospective cohort study based on the Chinese population. The study is expected to identify associations between GDM and the neurodevelopment of OGDM from infancy to preschool age and to further assess the effects of GDM on neurodevelopmental trajectories.

\section{METHODS AND ANALYSIS \\ Study design and patient recruitment}

This single-centre prospective cohort study is conducted in the First People's Hospital of Chongqing Liangjiang New Area, Chongqing, China. Participant recruitment for the study has began in June 2019 and is scheduled to be completed in Year 1.5-2 of the study and offspring will befollowed for 72 months. A total of 490 OGDM (GDM group) will be enrolled. and 490 offspring of healthy mothers (control group) will be enrolled in a 1:1 ratio

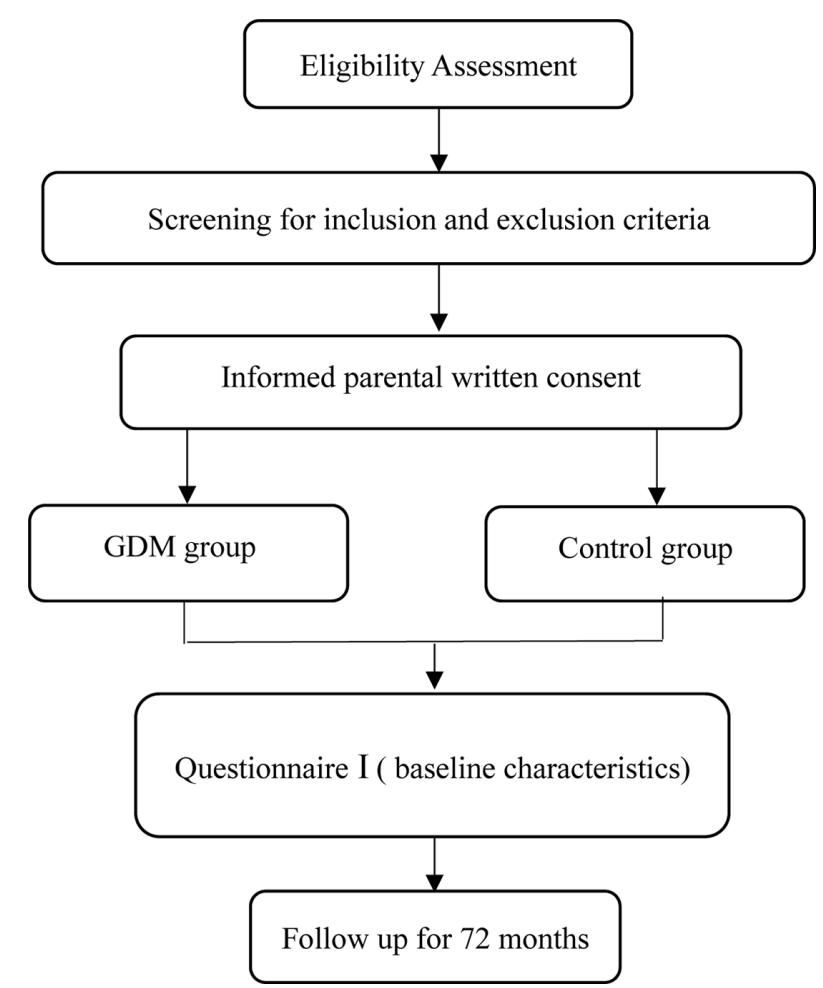

Figure 1 Flow diagram of the study protocol. GDM, gestational diabetes mellitus.

using the random sampling method within 3 days after the birth of each OGDM. The inclusion criteria for participants in this study are as follows: (1) Term infants; (2) Singleton birth; (3) Apgar scores after birth at $1 \mathrm{~min}$, 5 min and $10 \mathrm{~min} \geq 7$; (4) No other perinatal diseases that seriously affect growth and development; (5) No apparent birth defects and (6) No congenital or genetic conditions that cause developmental disability, such as hypothyroidism, phenylketonuria, toxopasma, rubellaVirus, cytomegalovirus and herpessimplxvirus (TORCH) infections, Down syndrome. The exclusion criteria: (1) Mothers with type 1 or type 2 diabetes prior to pregnancy; (2) Mothers with no oral glucose tolerance test (OGTT) diagnostic results; (3) Mothers with serious acute or chronic infectious diseases; (4) Mothers with other complications during pregnancy; (1)Mothers with a gestational age greater than 35 years; and (6) Children aged 0-6 years who suffer from diseases that seriously affect metabolism and growth and development. The flow diagram of the study protocol is described (figure 1).

\section{Patient and public involvement}

Patients have not been involved in the design of and recruitment for this study. The results of the study will be published through open access journals and made to the patients and the public.

\section{Study procedures}

All pregnant women who visit the outpatient obstetrics department on a regular basis and be given a 2-hour OGTT with $75 \mathrm{~g}$ glucose loads at 24-28 weeks of gestation. GDM is diagnosed according to The International Association 
of Diabetes and Pregnancy Study Groups (IADPSG) 2010 criteria: fasting glucose $\geq 5.1 \mathrm{mmol} / \mathrm{L}(92 \mathrm{mg} / \mathrm{dL})$, or 1-hour glucose $\geq 10.0 \mathrm{mmol} / \mathrm{L}(180 \mathrm{mg} / \mathrm{dL})$ or 2-hour glucose $\geq 8.5 \mathrm{mmol} / \mathrm{L}(153 \mathrm{mg} / \mathrm{dL}) .{ }^{16}$ After initial communication with the mothers within 48 hours of delivery, all mothers in both groups will voluntarily participate in this study and provide informed consent per the request of the ethics committee. At the same time, questionnaire I will be completed, which includes common items (age, height, weight, education level, average monthly household income, smoking and drinking habits and permanent residence of the parents), items related to the mothers (prepregnancy weight, pregnancy number, the number of births, pregnancy-related conditions, routine prenatal blood indicators and the mode of delivery) and items related to the offspring (sex, date of birth, birth weight, recumbent birth length, Apgar score and gestational weeks at birth). The two groups will be followed up at 1, 3, 6, 12, 24, 36, 48, 60 and 72 months of age. Developmental assessments, anthropometric data (recumbent length/height and weight), routine blood parameters (haemoglobin $(\mathrm{Hb})$, mean corpuscular volume (MCV), mean corpuscular $\mathrm{Hb}$ concentration (MCHC), red cell distribution width (RDW\%) and platelet count (PLT)), and questionnaire II results addressing average monthly household income, feeding patterns (breastfeeding, partial breastfeeding, formula feeding and an additional dietary survey after 6 months of age), and the main caretakers and illnesses of the offspring will be recorded at each follow-up time point.

Peabody Developmental Motor Scales 2 (PDMS-2) and the Gesell Development Inventory (GDI) will be used as standardised assessments to evaluate offspring at appropriate time points by professional appraisers who have received standardised training. We will implement standardised evaluation procedures and perform regular self-assessments to reduce errors among evaluators and maximise the accuracy and reliability of the results. During the assessments, the assessment environment will be quiet and comfortable and the professional appraisers and offspring will be allowed to adapt to each other. We will avoid evaluating offspring with illnesses, drowsiness and other non-compliant conditions. PDMS-2, as a developmental motor scale with high reliability and validity, will be selected to assess motor abilities at 1, 3 and 6 months old. ${ }^{17} 18$ The scale includes gross and fine motor assessment scales, which are used to evaluate offspring's motor development. The gross motor assessment scale includes 151 items to test the ability of reflexes, stationary subtests, locomotion, object manipulation and object control ( $>12$ months). The fine motion rating scale includes 98 items to test grasping ability and visual-motor integration. After the test, quotients (the gross motion quotient (GMQ), fine motion quotient (FMQ) and total motion quotient (TMQ) ) will be calculated by the original score. The score can be interpreted as follows: a quotient above 90 and below 110 is normal, the mean is 100 and the mean square error of the quotient is set as 15 . A quotient increase of
$1 \mathrm{SD}$ is above normal, an increase of $2 \mathrm{SD}$ is good and an increase of $3 \mathrm{SD}$ is excellent. Quotient decreases of $1 \mathrm{SD}, 2 \mathrm{SD}$ and $3 \mathrm{SD}$ are below normal, poor and very poor, respectively. ${ }^{19}$ The neuropsychological development of offspring will be evaluated at $6,12,24,36,48,60$ and 72 months old using the GDI. The GDI is accurate and comprehensive for the assessment of children and has relatively reliable diagnostic value. The GDI has been widely used internationally ${ }^{20}$ and objectively and comprehensively evaluates five skill areas: adaptive behaviour, gross motor, vision and fine motor skills, language and personal-social behaviour. ${ }^{21}$ The development quotient (DQ) is calculated by the original score for each of the skill areas. The results are expressed by three categories: normal, bradygenesis and marginal. A $D Q \geq 85$ is normal. A DQ $\leq 75$ reflects bradygenesis. A DQ between 75 and 85 is regarded as marginal. ${ }^{22}$

All offspring will be measured in a special room by trained nurses. The recumbent length and weight of offspring under 3 years old wearing underwear and no shoes will be measured with an infant precision examination instrument (TJ-120C, Shanghai, China), which has an accuracy of $(0.01 \mathrm{~kg}, 1 \mathrm{~mm})$. A child standing precision examination instrument (JT-220B, Shanghai, China) with a range up to $150 \mathrm{~cm}$ and an accuracy of $(0.1 \mathrm{~kg}, 1 \mathrm{~mm})$ will be used to measure length (standing) and weight from 3 years old to 6 years old. All values will be measured three consecutive times and averaged. We will calculate corresponding length-for-age z-scores (LAZ), weightfor-age z-scores (WAZ), weight-for-length z-scores (WLZ, not calculated after 60 months of age) and BMI-for-age z-scores (BMIZ) from 1 month to 72 months of age using the WHO 2011 V.3.2.2 software according to the WHO Growth Curve Standards of 2006. At different follow-up times, trained, proficient staff will collect data and input them into an electronic system. The follow-up schedule is showed in table 1

\section{Follow-up protocol}

The two groups will be followed for 72 months at the child healthcare department of the designated hospital to complete developmental assessments, anthropometric measurements, blood tests and questionnaires at 1, 3, 6, 12, 24, 36, 48, 60 and 72 months old. In this study, the follow-up time is relatively long. To improve the follow-up rate, a detailed quality control plan has been formulated as follows: (1) We have carried out unified training, established a follow-up system, ensured the clarity of the follow-up work content, workflows, and service standards for specially assigned persons, and improved the awareness of follow-up work performed by specially assigned persons. (2) We have created a template for children's health education, finalised and printed it for parents to read and educated the parents of the offspring in the two groups in the hospital. The publicity and educational content included the study content, follow-up schedule, feeding guidance and so on. The purpose is to improve the follow-up compliance of the parents and inform the 


\begin{tabular}{|c|c|c|c|c|c|c|c|c|c|c|}
\hline & \multicolumn{10}{|c|}{ Follow-up } \\
\hline & $\begin{array}{c}0 \\
\text { month }\end{array}$ & $\begin{array}{c}1 \\
\text { month }\end{array}$ & $\begin{array}{c}3 \\
\text { months }\end{array}$ & $\begin{array}{c}6 \\
\text { months }\end{array}$ & $\begin{array}{c}12 \\
\text { months }\end{array}$ & $\begin{array}{c}24 \\
\text { months }\end{array}$ & $\begin{array}{c}36 \\
\text { months }\end{array}$ & $\begin{array}{c}48 \\
\text { months }\end{array}$ & $\begin{array}{c}60 \\
\text { months }\end{array}$ & $\begin{array}{c}72 \\
\text { months }\end{array}$ \\
\hline Questionnaire I & $\times$ & & & & & & & & & \\
\hline Questionnaire II & & $\times$ & $\times$ & $x$ & $\times$ & $\times$ & $\times$ & $x$ & $\times$ & $\times$ \\
\hline PDMS-2 & & $x$ & $\times$ & $x$ & & & & & & \\
\hline Gesell Development Inventory & & & & $x$ & $\times$ & $\times$ & $\times$ & $x$ & $\times$ & $x$ \\
\hline Anthropometric measurements & & $x$ & $\times$ & $\times$ & $x$ & $\times$ & $x$ & $x$ & $x$ & $x$ \\
\hline Routine blood examinations & & $x$ & $x$ & $x$ & $x$ & $x$ & $x$ & $x$ & $x$ & $x$ \\
\hline
\end{tabular}

PDMS-2, Peabody Developmental Motor Scales 2.

parents of the department's consultation phone number, email and WeChat information such that parents can contact us at any time when they have questions. We have also held regular popular science lectures to build good trust relationships with the parents. (3) We have created a follow-up registration form and clarified the follow-up content to ensure an understanding of the recent situation of the offspring and remind the parents to arrive at the hospital on time. (4) We have conducted call visits. The offspring in the two groups have been followed by specially assigned persons. A telephone follow-up area equipped with special telephones and computers has been established. The environment of the follow-up area is quiet and convenient for the specially assigned persons to arrange telephone follow-up work on their own time. During the follow-up process, information on the offspring can be accessed at any time and personalised education and interactions can be carried out for parents. A procedure for telephone follow-ups has been established for each department. Each follow-up contact includes six steps: confirmation of the honorific name, self-introduction, purpose explanation, care and inquiry, explanation and announcement, and inquiry demand and appreciation. (5) When the parents do not answer the phone, we will make contact through WeChat or email. Moreover, we have regularly distributed popular science articles via WeChat or email.

\section{Outcomes}

\section{Primary outcome}

The primary outcomes of this study are the cross-sectional developmental assessment results (the quotients of PDMS-2 (GMQ, FMQ and TMQ) and the DQ of the GDI) of the OGDM and offspring of non-GDM mothers, which were used to identify the associations between GDM and the offspring's neurodevelopment from 1 to 72 months. In addition, we will compare longitudinal data to further assess the effects of GDM on neurodevelopmental trajectories.

\section{Secondary outcomes}

We will analyse z-scores (LAZ, WAZ, WLZ and BMIZ) to identify the associations between GDM and the offspring's growth and to longitudinally assess the effects of GDM on growth trajectories from 1 to 72 months.

Moreover, this study will investigate changes in routine blood indicators, including the $\mathrm{Hb}, \mathrm{MCV}, \mathrm{MCHC}, \mathrm{RDW} \%$ and PLT of the two groups at each follow-up time.

\section{Sample size calculation}

The sample size is difficult to calculate because the incidence of neurodevelopmental abnormalities in offspring exposed to GDM has not been established. We considered the results of a small number of previous studies performed in a similar population to calculate the required sample size. ${ }^{23-25}$ Considering an alpha error rate of $0.05\left(\alpha=0.05, \mathrm{Z}_{\alpha / 2}=1.96\right)$, a power of $80 \%(\beta=0.2$, $\mathrm{Z}_{\beta}=0.842$ ), $\mathrm{r}=1, p_{0}$ (prevalence of neurodevelopmental abnormalities in the non-exposed group $)=4 \%$ and $p_{1}$ (prevalence of neurodevelopmental abnormalities in the exposure group) $=8.8 \%$, and a calculated effective sample size is 409 and assuming a loss to follow-up rate of $15 \%-20 \%$, the totalsample size for this study is as follows: GDM group $=490$ and control group $=490$.

$$
N_{\text {keley }}=\frac{\left(\frac{z \alpha}{2}+z_{\beta}\right)^{2} P(1-P)(\gamma+1)}{\gamma\left(P_{0}-P_{1}\right)^{2}} P=\frac{p_{0}+\gamma p_{1}}{\gamma+1}
$$

\section{Statistical analyses}

Data will be analysed by SPSS V.19.0 . For normally distributed data, continuous variables will be represented by means and analysed by t-test. The values are expressed as the median for abnormally distributed data and compared using non-parametric tests. Categorical variables of the two groups will be analysed by the $\chi^{2}$ test. We will construct linear growth models to assess the neurodevelopmental trajectories and growth trajectories. In addition, multiple linear regression will be used to analyse confounders, such as age, height, weight, education level, average monthly household income, smoking and drinking habits of the parents. $\mathrm{p}<0.05$ will be considered indicative of a significant difference. For lost to follow-up data, we will use the multiple filling method .

\section{Ethics and dissemination}

This prospective cohort study has been approved by the Ethics Committee of the Children's Hospital of 
Chongqing Medical University (Approval Number: (2019) Institutional Review Board (IRB) (STUDY) No. 85). Before entering the study, written informed consent will be obtained from the parents. The findings of this study will be disseminated through open access journals, peer-reviewed journals and conference presentations.

\section{DISCUSSION}

With rapid development in China, changes in lifestyle, the implementation of the second-child policy, and increases of older people and overweight parturient women, the incidence of GDM continues to increase.

Studies have shown that intrauterine exposure to GDM is a risk factor for the short-term health and long-term higher BMI of offspring. ${ }^{26-29}$ However, few studies have discussed the associations between intrauterine exposure to GDM and the neurodevelopmental of offspring at different ages, and existing conclusions are controversial. Among studies on the associations between GDM and the neurodevelopmental of offspring, Fraser et al have found negative associations: OGDM have lower average scores in 9-year compulsory education among their non-compatriots, lower IQ scores at the age of 18 when they take a compulsory recruitment test, ${ }^{30}$ and impaired language expression in middle childhood compared with the control group. ${ }^{25}$ In addition, a cohort study in Israeli has reported that the incidence of neuropsychiatric morbidity in the OGDM is higher than control. In contrast, Ornoy has found a null association between GDM and the cognitive ability of offspring. ${ }^{31}$ A mother-child cohort study in Greece have showed that GDM do not affect the neurodevelopment of offspring. Townsend have showed that the offspring of diabetic mothers perform as well as the controls in neurobehavioral tests, although the study does not control for potential confounders, such as socioeconomic status and pre-pregnancy BMI. ${ }^{32}$ However, most of the studies are cross-sectional at specific time points and few studies have focused on the neurodevelopmental patterns of OGDM from infancy to preschool age.

This single-centre prospective cohort study tracks the growth and development of OGDM from 1 month to 72 months in China. This study not only identifies associations between GDM and the neurodevelopment of offspring through a cross-sectional comparison but also analyses the effects of GDM on neurodevelopmental trajectories through a longitudinal comparison. In the whole research process, we will use standardised anthropometric methods and developmental assessment tools and fully consider potential confounders. This study has several limitations. First, we have calculated the sample size based on existing study results, although the incidence of GDM causing differences in the neurodevelopmental of offspring is still unclear, which may lead to inaccurate calculations of sample size; thus, the associations between GDM and the neurodevelopmental of offspring therefore cannot be clarified. If this is the case, we will expand the sample size. Second, in this study, the follow-up time is relatively long, which may lead to a higher lost to follow-up rate. We have formulated a complete follow-up quality control plan before the study to minimise lost to follow-up.

In summary, the conclusions of this study may reveal the neurodevelopmental trends and trajectory of OGDM and provide a more accurate assessment of the neurodevelopmental status of offspring at each time point such that we can implement timely interventions to prevent or reverse adverse outcomes. In addition, the conclusions may be helpful for health departments responsible for the health of mothers and their children with gestational diabetes and facilitate improvement of the standardised management of gestational diabetes. The study may also provide references for formulating healthcare strategies during pregnancy and further follow-up monitoring of offspring's health.

\section{Author affiliations}

${ }^{1}$ Growth, Development and Mental Health Center of Children and Adolescents, Children's Hospital of Chongqing Medical University; National Clinical Research Center for Child Health and Disorders; Ministry of Education Key Laboratory of Child Development and Disorders; Chongqing Key Laboratory of Child Health and Nutrition, Chongqing, China

${ }^{2}$ Department of Child Care, The First People's Hospital of Chongqing Liangjiang New Area, Chongqing, China

${ }^{3}$ Department of Health Statistics, Chongqing Medical University, Chongqing, China ${ }^{4}$ Department of Outpatient, The First People's Hospital of Chongqing Liangjiang New Area, Chongqing, China

${ }^{5}$ Department of Obstetrics, The First People's Hospital of Chongqing Liangjiang New Area, Chongqing, China

Acknowledgements We thank everyone who contributed to this study, including the parents of the children in the two groups and colleagues responsible for the recruitment, evaluation, follow-up and management.

Contributors LC and CL have wrote the initial study protocol. CL, TY, JC and TL have participated in revising and amending the protocol. LC, PZ, YC, YoL, YiL, YH, $\mathrm{ZW}, \mathrm{HP}$ and $\mathrm{YZ}$ have participated in the recruitment for this study. CL, PZ, YC, YoL, YF and $\mathrm{YZ}$ will collect the data. $\mathrm{LC}$ and $\mathrm{BP}$ will analyse the data. All authors have read and approved the final version.

Funding This study is supported by the Scientific Research Projects unit of Chongqing (project number: 2019QNXM035, cstc2018jscX-mszdX0023) and Open Project Fund from Key Laboretory of Reproduction Regulation of NHC(project number: KF2018-03).

Competing interests None declared.

Patient and public involvement Patients and/or the public were not involved in the design, or conduct, or reporting, or dissemination plans of this research.

Patient consent for publication Not required.

Provenance and peer review Not commissioned; externally peer reviewed.

Open access This is an open access article distributed in accordance with the Creative Commons Attribution Non Commercial (CC BY-NC 4.0) license, which permits others to distribute, remix, adapt, build upon this work non-commercially, and license their derivative works on different terms, provided the original work is properly cited, appropriate credit is given, any changes made indicated, and the use is non-commercial. See: http://creativecommons.org/licenses/by-nc/4.0/.

ORCID iDs

Chao Li http://orcid.org/0000-0003-2596-3754

Li Chen http://orcid.org/0000-0002-2614-1528

\section{REFERENCES}

1 Baz B, Riveline J-P, Gautier J-F. Endocrinology of pregnancy: gestational diabetes mellitus: definition, aetiological and clinical aspects. Eur J Endocrinol 2016;174:R43-51. 
2 Cho NH, Shaw JE, Karuranga S, et al. IDF diabetes atlas: global estimates of diabetes prevalence for 2017 and projections for 2045. Diabetes Res Clin Pract 2018;138:271-81.

3 Gao C, Sun X, Lu L, et al. Prevalence of gestational diabetes mellitus in mainland China: A systematic review and meta-analysis. $J$ Diabetes Investig 2019;10:154-62.

4 Hanson M. The birth and future health of DOHaD. J Dev Orig Health Dis 2015;6:434-7.

5 Richter LM, Daelmans B, Lombardi J, et al. Investing in the foundation of sustainable development: pathways to scale up for early childhood development. Lancet 2017;389:103-18.

6 Meur S, Mann NP. Infant outcomes following diabetic pregnancies. Paediatr Child Health 2007;17:217-22.

7 Tam WH, Ma RCW, Ozaki R, et al. In utero exposure to maternal hyperglycemia increases childhood cardiometabolic risk in offspring. Diabetes Care 2017;40:679-86.

8 Hillier TA, Pedula KL, Vesco KK, et al. Impact of maternal glucose and gestational weight gain on child obesity over the first decade of life in normal birth weight infants. Matern Child Health $J$ 2016;20:1559-68.

9 Crume TL, Ogden L, Daniels S, et al. The impact of in utero exposure to diabetes on childhood body mass index growth trajectories: the epoch study. J Pediatr 2011;158:941-6.

10 Veena SR, Krishnaveni GV, Srinivasan K, et al. Childhood cognitive ability: relationship to gestational diabetes mellitus in India. Diabetologia 2010;53:2134-8.

11 Dahlquist G, Källén B. School marks for Swedish children whose mothers had diabetes during pregnancy: a population-based study. Diabetologia 2007;50:1826-31.

12 Yamashita Y, Kawano Y, Kuriya N, et al. Intellectual development of offspring of diabetic mothers. Acta Paediatr 1996;85:1192-6.

13 DeBoer T, Wewerka S, Bauer PJ, et al. Explicit memory performance in infants of diabetic mothers at 1 year of age. Dev Med Child Neurol 2005:47:525-31.

14 Xiang $\mathrm{AH}$, Wang $\mathrm{X}$, Martinez MP, et al. Maternal gestational diabetes mellitus, type 1 diabetes, and type 2 diabetes during pregnancy and risk of ADHD in offspring. Diabetes Care 2018;41:2502-8.

15 Bonilla C, Lawlor DA, Ben-Shlomo Y, et al. Maternal and offspring fasting glucose and type 2 diabetes-associated genetic variants and cognitive function at age 8: a Mendelian randomization study in the Avon longitudinal study of parents and children. BMC Med Genet 2012;13:90.

16 Metzger BE, Gabbe SG, Persson B, et al. International association of diabetes and pregnancy study groups recommendations on the diagnosis and classification of hyperglycemia in pregnancy: response to Weinert. Diabetes Care 2010;33:e98-82.

17 Morgan C, Novak I, Dale RC, et al. Optimising motor learning in infants at high risk of cerebral palsy: a pilot study. BMC Pediatr 2015;15:30.

18 Tavasoli A, Aliabadi F, Eftekhari R. Motor developmental status of moderately low birth weight preterm infants. Iran J Pediatr 2014;24:581.

19 Folio MR, Fewell R. Peabody developmental motor scales: examiner's manual. 2 edn. Austin: PROED, 2000.

20 Song J, Zhu YM. Children's neuropsychological ests. Shanghai, China: Shanghai Scientific and Technological Publishing Company, 1987.

21 Zhu Y, Lu S, Tang C, et al. Application of DDST in China: retrospective and prospective era. J Clin Pediatr 1983;1:129-32.

22 Cohen LG, Spenciner LJ. Assessment of young children. Longman Pub Group, 1994.

23 Shriberg LD, Tomblin JB, McSweeny JL. Prevalence of speech delay in 6-year-old children and comorbidity with language impairment. $J$ Speech Lang Hear Res 1999;42:1461-81.

24 Silva PA. The prevalence, stability and significance of developmental language delay in preschool children. Dev Med Child Neurol 1980;22:768-77.

25 Dionne G, Boivin M, Séguin JR, et al. Gestational diabetes hinders language development in offspring. Pediatrics 2008;122:e1073-9.

26 Jones CW. Gestational diabetes and its impact on the neonate. Neonatal Netw 2001;20:17-23.

27 Catalano PM, Mclntyre HD, Cruickshank JK, et al. The hyperglycemia and adverse pregnancy outcome study: associations of GDM and obesity with pregnancy outcomes. Diabetes Care 2012;35:780-6.

28 Silverman BL, Rizzo TA, Cho NH, et al. Long-Term effects of the intrauterine environment. The northwestern university diabetes in pregnancy center. Diabetes Care 1998;21 Suppl 2:B142.

29 Hammoud NM, Visser GHA, van Rossem L, et al. Long-term BMl and growth profiles in offspring of women with gestational diabetes. Diabetologia 2018;61:1037-45.

30 Fraser A, Almqvist C, Larsson $\mathrm{H}$, et al. Maternal diabetes in pregnancy and offspring cognitive ability: sibling study with 723,775 men from 579,857 families. Diabetologia 2014;57:102-9.

31 Ornoy A. Growth and neurodevelopmental outcome of children born to mothers with pregestational and gestational diabetes. Pediatr Endocrinol Rev 2005;3:104-13.

32 Townsend EL, Georgieff MK, Nelson CA. Neurobehavioral functioning in five-year-old children born to diabetic mothers. Cogniție Creier Comportament, 2005. 